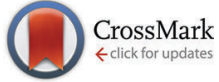

Cite this: DOI: 10.1039/c5sm01580d

\title{
Dimensional reduction of duplex DNA under confinement to nanofluidic slits
}

\author{
Fernando Vargas-Lara, ${ }^{\star a}$ Samuel M. Stavis, ${ }^{b}$ Elizabeth A. Strychalski, ${ }^{c}$ \\ Brian J. Nablo, ${ }^{d}$ Jon Geist, ${ }^{d}$ Francis W. Starr ${ }^{e}$ and Jack F. Douglas ${ }^{* a}$
}

There has been much interest in the dimensional properties of double-stranded DNA (dsDNA) confined to nanoscale environments as a problem of fundamental importance in both biological and technological fields. This has led to a series of measurements by fluorescence microscopy of single dsDNA molecules under confinement to nanofluidic slits. Despite the efforts expended on such experiments and the corresponding theory and simulations of confined polymers, a consistent description of changes of the radius of gyration of dsDNA under strong confinement has not yet emerged. Here, we perform molecular dynamics (MD) simulations to identify relevant factors that might account for this inconsistency. Our simulations indicate a significant amplification of excluded volume interactions under confinement at the nanoscale due to the reduction of the effective dimensionality of the system. Thus, any factor influencing the excluded volume interaction of dsDNA, such as ionic strength, solution chemistry, and even fluorescent labels, can greatly influence the dsDNA size under strong confinement. These factors, which are normally less important in bulk solutions of dsDNA at moderate ionic strengths because of the relative weakness of the excluded volume interaction, must therefore be under tight control to achieve reproducible measurements of dsDNA under conditions of dimensional reduction. By simulating semi-flexible polymers over a range of parameter values relevant to the experimental systems and exploiting past theoretical treatments of the dimensional variation of swelling exponents and prefactors, we have developed a novel predictive relationship for the in-plane radius of gyration of long semi-flexible polymers under slit-like confinement. Importantly, these analytic expressions allow us to estimate the properties of dsDNA for the experimentally and biologically relevant range of contour lengths that is not

currently accessible by state-of-the-art MD simulations.

Received 26th June 2015, Accepted 18th August 2015

DOI: $10.1039 / c 5 s m 01580 d$

www.rsc.org/softmatter
An understanding of the many factors influencing the size of dsDNA under nanoscale confinement is highly relevant to rationally designing measurement technologies for genomic sequencing and medical sensing and for accurately describing crowding effects on dsDNA organization in living systems. Consequently, there have been many recent theoretical and experimental studies of this phenomenon. ${ }^{1-15}$ In particular, it has been possible for two decades to image individual fluorescently labeled dsDNA under conditions of nanoscale confinement in

\footnotetext{
${ }^{a}$ Materials Science and Engineering Division, National Institute of Standards and Technology, Gaithersburg, MD, 20899, USA. E-mail: luis.vargas@nist.gov, jdouglas@nist.gov

${ }^{b}$ Center for Nanoscale Science and Technology, National Institute of Standards and Technology, Gaithersburg, MD, 20899, USA

${ }^{c}$ Biosystems and Biomaterials Division, National Institute of Standards and Technology, Gaithersburg, MD, 20899, USA

${ }^{d}$ Semiconductor and Dimensional Metrology Division, National Institute of Standards and Technology, Gaithersburg, MD, 20899, USA

${ }^{e}$ Department of Physics and Department of Molecular Biology and Biochemistry, Wesleyan University, Middletown, CT, 06459, USA
}

nanofluidic devices with slit-like geometries. In this way, the in-plane radius of gyration of dsDNA as a function of slit height has been recently measured. Fig. 1 summarizes the results of these experimental measurements, which exhibit a puzzling scatter under nominally similar experimental conditions. Scaling arguments and continuum chain models have dominated the majority of the recent theoretical treatments of this problem, which generally ignore polymer-surface interactions and have the more serious problem of a breakdown of the continuum limit under strong confinement. ${ }^{16}$ It should then come as no surprise that these theoretical treatments are inadequate to describe dsDNA under such conditions.

Here, we perform molecular dynamics (MD) simulations of a simple model of double-stranded DNA (dsDNA) that incorporates excluded volume, polymer-surface, and polymer-polymer interactions. Our simulations indicate that the largest underappreciated effect in previous modeling is the excluded volume interaction, which becomes amplified under conditions of strong confinement due to the reduction of the effective spatial dimensionality. In addition, attractive polymer-polymer and 

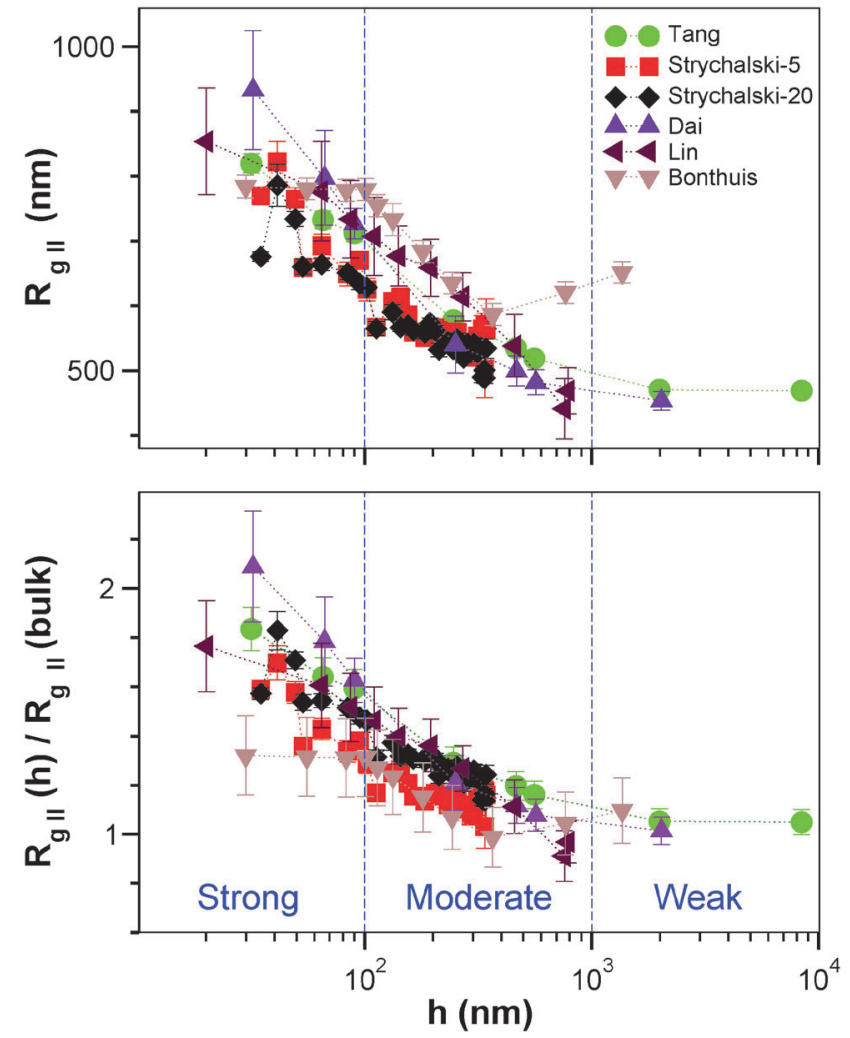

Fig. 1 The upper panel compares experimental measurements and inferences of the size of dsDNA under confinement to nanofluidic slits; Tang et $a l^{8}{ }^{8}$ (in green circles), Strychalski et al., ${ }^{5,11}$ (5:1 in red squares, $20: 1$ in black diamonds), Dai et al..$^{12}$ (purple upright triangles), and Lin et al. ${ }^{13}$ (maroon sideways triangles), Bonthuis et al. ${ }^{6}$ (brown inverted triangles). In these experimental measurements, the relevant metric of dsDNA size is the most probable (mode) value of the component of the radius of gyration parallel to the slit, abbreviated as $R_{\mathrm{g} \|}$. The lower panel presents this in-plane size of dsDNA under slit-like confinement, normalized by estimated values of the size of dsDNA in bulk solution. This normalization contributes additional experimental uncertainty. For these experimental systems, the transition between strong and moderate confinement occurs around slit heights $h$ that are twice the dsDNA persistence length $2 l_{\mathrm{p}}, h \approx 100 \mathrm{~nm}$, while the transition between moderate and weak confinement occurs around slit heights around, $h \approx 1000 \mathrm{~nm}$. Dashed lines guide the eyes.

polymer-surface interactions appreciably influence the polymer size. These results provide new insight into this complex system, which is essential for a meaningful comparison with experimental measurements and theoretical predictions.

We organize our paper as follows. In Section 1, we briefly review the experimental and theoretical situation to clearly define the scientific problem and the interactions and properties that are relevant to our calculations. In Section 2, we describe a molecular model of dsDNA as a semi-flexible polymer having fixed persistence length and variable effective diameter, given the range of suggested values for the effective diameter. In Section 3, we use this model to perform MD simulations over a wide range of values of slit height, contour length, and effective diameter, and we deduce suitable mathematical approximants for the polymer dimensions in terms of these basic variables. Additionally, we perform MD simulations that include attractive polymer-surface and polymer-polymer interactions to assess the qualitative importance of these interactions on polymer size. In Section 4, we directly compare our computational results with the experimental measurements described in Section 1. In this comparison, we find good agreement for a range of effective diameters that are fully consistent with standard estimates from molecular biology. This comparison also indicates that inadvertent variation in the excluded volume interaction, which is less significant for dsDNA in bulk solution than under confinement, probably contributes to the variability of the experimental measurements. We also discuss details of the experimental systems to clarify uncertainties in both the experimental measurements and input parameters for models of dsDNA molecules under confinement. We conclude in Section 5.

\section{Brief review of previous studies of polymers under confinement to slits}

We begin by giving a brief overview of recent experimental studies of dsDNA under confinement to nanofluidic slits. Fig. 1 compares the results of several experimental measurements and inferences, and Table 1 presents measurement parameters of the experimental systems. The upper panel of Fig. 1 summarizes single-molecule measurements of the in-plane radius of gyration $R_{\mathrm{g} \|}$ of dsDNA obtained from references. ${ }^{3,5,6,8,11-13}$ The lower panel of Fig. 1 presents the in-plane size of the dsDNA normalized by an estimate of the most probable (mode) value in bulk solution $R_{\mathrm{g} \|}$ (bulk). Although this dimensionless measure of dsDNA size in terms of the chain size in bulk solution is theoretically attractive, there are significant uncertainties in estimating $R_{\mathrm{g} \|}$ (bulk) that compound experimental uncertainties in estimating $R_{\mathrm{g} \|}(h)$ under confinement. In addition, there are other factors that have not been tightly controlled or precisely characterized that might account for the variability of the experimental results, as well as the proposed models of dsDNA under confinement. For example, there has been little attention given to the dimensional effects of strong slit-like confinement on excluded volume interactions of long semi-flexible polymers or the effects of solution conditions on the balance of attractive polymer-polymer and excluded volume interactions. Moreover, previous studies have scarcely considered the influence of polymer-surface interactions in strong slit-like confinement on the size of semi-flexible polymers. Considering these issues, the inconsistency of the measurements and predictions is perhaps not surprising.

Scaling arguments have long been applied to predict the dimensions of polymers under confinement. ${ }^{19}$ The classic work of Daoud and de Gennes ${ }^{20}$ considered the case of a swollen flexible polymer, modeled as a self-avoiding walk confined between repulsive walls. This scaling argument predicts that the size of a swollen polymer confined to such a slit scales as $R_{\mathrm{g}} \sim M^{3 / 4}\left(h / l_{\mathrm{p}}\right)^{-1 / 4}$, where $M$ is the polymer mass, $h$ is the slit height, and $l_{\mathrm{p}}$ is the polymer persistence length. This scaling argument for $R_{\mathrm{g}}$ has been highly influential in experimental investigations of confined polymers, but Odijk questioned the applicability of this argument in the strong confinement regime, 


\section{Minimal model for computation}

The formulation of a predictive model for this system requires the introduction of a theoretical framework to guide the organization of simulation results into useful mathematical approximants. To proceed, we must recognize that we confront two fundamentally difficult problems - the problem of polymer excluded volume interactions and the inherent problem of describing strong confinement based on a continuum theory for which the confinement scale is on the order of the coarsegraining scale of the model. These two problems are coupled for confined polymers, which demands a numerical rather than an analytical treatment. Even the most powerful analytical method, such as renormalization group theory, ${ }^{22}$ cannot help us with solving the problem of dimensional reduction, as Douglas and Freed $^{16}$ discussed in the related context of the adsorption of a flexible end-tethered polymer onto a planar surface. In particular, Douglas and Freed solved the problem of the adsorption of a single random walk polymer onto a planar surface based on both continuum and discrete lattice models. In these models, the confinement derives from the polymer-surface interaction, which localizes the polymer to an interfacial region if the attractive interaction is sufficiently large to adsorb the polymer. The associated localization length of the adsorbed polymer is analogous to slit height for polymers between two non-adsorbing surfaces. An exact calculation based on the continuum polymer model, described as a Gaussian coil polymer, as a function of an increasingly attractive polymer-surface interaction corresponding to confinement, was inconsistent with the corresponding exact lattice random walk calculation for the in-plane size of an ideal polymer.

Douglas and Freed identified the discrepancy between these formally equivalent calculations as the breakdown of the continuum limit on which the conventional Gaussian polymer model is based. The same difficulty must arise in the wormlike chain model, which does not account for excluded volume interactions. Actually, this is a problem for any field theory or continuum polymer model under strong confinement. Douglas and Freed, however, were able to offer a practical solution to 
this problem of dimensional reduction by demanding the equality of the results obtained from the continuum and lattice-based models. This consistency criterion requires that the effective spatial dimensionality becomes a function of the geometrical confinement. This is an effect that we must incorporate in our modeling of confined dsDNA.

The change in size of ideal polymers in the absence of excluded volume interactions under strong confinement is relatively small. In Section 3.2, we briefly consider such a model in comparison to polymers with excluded volume interactions, which show a much larger change in size, because the swelling exponent $\nu$ governing the mass $M$ scaling of the mean size of the polymer, $R_{\mathrm{g}} \sim M^{\nu}$, depends strongly on the spatial dimension; $\nu=0.58-0.59$ for threedimensional self-avoiding polymers and $\nu=0.75$ for twodimensional self-avoiding polymers. $\dagger^{23-27}$ A change in $\nu$ as a result of dimensional reduction must then translate into a significant change in molecular size if the polymers are not perfectly ideal. Our predictive model must take into account this effect of dimensional reduction on not only the swelling exponent $\nu$, but also on the prefactor $A$, in the relation $R_{\mathrm{g}}=A M^{\nu}$. Both model parameters are then relevant metrics of dimensional reduction. Exact enumeration $^{26}$ and renormalization group theory ${ }^{22}$ provide guidance in the limits of three dimensions and two dimensions for comparison to our calculated values of $\nu$ and $A$ describing the results of dimensional reduction.

\subsection{Coarse-grained model of dsDNA under confinement to nanofluidic slits}

We model the dsDNA as a chain of connected beads ${ }^{29}$ interacting through a shifted 12-6 Lennard-Jones potential $U_{\mathrm{LJ}}^{12-6}$,

$$
U_{\mathrm{LJ}}^{12-6}(r)=4 \varepsilon\left[\left(\frac{\sigma}{r-\Delta}\right)^{12}-\left(\frac{\sigma}{r-\Delta}\right)^{6}\right] \quad r<r_{\mathrm{c}}+\Delta,
$$

where $\sigma$ and $\varepsilon$ are the Lennard-Jones length and energy parameters, respectively, $\Delta$ is a shift factor that controls the bead size, and $r_{\mathrm{c}}$ is a cutoff distance. We use $r_{\mathrm{c}}=2^{\frac{1}{6}} \sigma$ for steric bead-bead interactions, or $r_{\mathrm{c}}=2.5 \sigma$ to include an attractive interaction of strength $\varepsilon$ among the beads that form the chain. To connect the beads along the chain, we add a Lennard-Jones potential, shifted by $\varepsilon$ and truncated at $r_{\mathrm{c}}=2^{\frac{1}{6}} \sigma$, to a finitely extensible, nonlinear elastic (FENE) anharmonic-spring potential,

$$
U_{\text {bond }}(r)=U_{\mathrm{LJ}}^{12-6}(r)+\varepsilon+U_{\mathrm{FENE}}(r),
$$

where,

$$
U_{\mathrm{FENE}}(r)=-\frac{k R_{0}^{2}}{2} \ln \left[1-\left(\frac{r-\Delta}{R_{0}}\right)^{2}\right]
$$

\footnotetext{
$\dagger$ The swelling exponent $\nu$ in two dimensions is equal to 0.75 for excluded volume interactions between nearest neighbors, but we expect the interaction range to influence the value of $\nu$, as in the case of spin models in two dimensions. For example, in the case of the closely related 8-vertex model, an extension of the Ising model that includes both the nearest- and next nearest-neighbor interactions, $\nu$ depends continuously on the ratio of the strength of these interactions. ${ }^{28}$ Douglas et al. discuss the $\nu$ estimates for self-interacting polymers in quasi-two dimensions. ${ }^{25}$
}

Here, $k=30 \varepsilon / \sigma^{2}$ is the bond strength, and $R_{0}=1.5 \sigma$ yields a maximum bond length $=1.5 \sigma+\Delta$. In this way, the shape of the bonding potential $U_{\text {bond }}(r)$ for all the effective diameters is the same, but we translate the bonding potentials by $\Delta$ along the bond axis.

We model the chain stiffness by a three-body bending potential,

$$
U_{\text {bend }}(\gamma)=k_{\text {bend }}(1-\cos \gamma),
$$

where $k_{\text {bend }}$ defines the bending constant of the chain, and $\gamma$ is the angle formed by three consecutive beads along the chain. To obtain the persistence length $l_{\mathrm{p}}$ of the chain, we compute the orientational correlation function of the beads from the scalar product of two unitary vectors $\left\langle\overrightarrow{u_{i}} \cdot \overrightarrow{u_{j}}\right\rangle$ where $\overrightarrow{u_{i}}$ is defined by the bond connectivity bases $i$ and $i+1$. The orientational correlation function decays with increasing $|i-j|$ and we identify the persistence length $l_{\mathrm{p}}$ as the length where $\overrightarrow{u_{i}} \cdot \overrightarrow{u_{j}}=1 / e$.

The chain interacts with the surfaces of the confining slit by a 9-3 Lennard-Jones potential $U_{\mathrm{LJ}}^{9-3}$ :

$$
U_{\mathrm{LJ}}^{9-3}(r)=\varepsilon_{\mathrm{S}}\left[\frac{2}{15}\left(\frac{\sigma}{r-\Delta}\right)^{9}-\left(\frac{\sigma}{r-\Delta}\right)^{3}\right] \quad r<r_{\mathrm{c}}+\Delta .
$$

For this potential, we take $r_{\mathrm{c}}=(2 / 5)^{\frac{1}{6}} \sigma$ or $r_{\mathrm{c}}=2.5 \sigma$ for repulsive or attractive interactions, respectively, and consider $\varepsilon_{\mathrm{s}}$ as a measure of polymer-surface attractive interaction. We generate 10 different initial configurations for every chain with a given effective diameter $d$, contour length $L$, or slit height $h$. We allow each chain to reach thermal equilibrium at temperature $T=1.0$ by performing MD simulations for $10^{7}$ time steps, after which we compute the dimensional properties for $10^{7}$ different chain configurations. We perform all simulations using the Large-scale Atomic Molecular Massively Parallel Simulator (LAMMPS). ${ }^{30}$ We fit simulation results to theoretical and empirical models using non-linear least-squares estimation implemented by the Levenberg-Marquardt algorithm. We report uncertainties as one standard deviation. Figures show uncertainty only if its value is larger than the data point.

\subsection{Selection of input parameters}

The predictive utility of any model depends on a proper selection of input parameters. For our model, the persistence length $l_{\mathrm{p}}$, effective diameter $d$, and contour length $L$ of the chain, as well as the slit height $h$, minimally describe the experimental systems discussed in Section 1. In addition, we vary the strength of the polymer-surface and polymer-polymer interactions to qualitatively explore the effect on $R_{\mathrm{g} \|}$. The values of these input parameters are more or less certain, motivating a brief discussion here and extended discussions in following sections.

Duplex DNA is a polyelectrolyte, having electrostatic interactions that can influence the dimensions of the polymer, depending on the ionic strength of the solution. ${ }^{31}$ The value of $l_{\mathrm{p}}$ is relatively certain at the experimental values of ionic strength, however, which effectively screen charge interactions. We fix the persistence length to a nominal value of $l_{\mathrm{p}} \approx 54 \mathrm{~nm}^{8}$ in all of our simulations. 
In contrast, the effective diameter of dsDNA is relatively uncertain in magnitude, as we discuss in detail in Section 4. Therefore, we consider a wide range of values of $d$ in our simulations. In particular, we vary $\Delta=-0.75,-0.5,0$, or 1 corresponding to chains having $d=(1.55,3.1,6.2$, or 12.4$) \mathrm{nm}$. Through a subsequent comparison of computational results to experimental measurements, we infer that $d$ has a value which is consistent with the standard estimate from molecular biology, $d=2.4 \mathrm{~nm}$.

To make this comparison, we take $L=24.3 \mu \mathrm{m}$ as a representative value of the contour length. This assumes a nominal dsDNA-dye stoichiometry and ignores experimental uncertainties associated with the number of fluorescent dye molecules. The use of fluorescent dye also potentially affects the persistence length and the effective diameter. We discuss these issues in Section 4.

For reference, we first establish the behavior of our model with different effective diameters in bulk solution. We then extend our simulations to the same model under confinement to a slit, with the slit height $h$ varying from regimes of strong, $h<2 l_{\mathrm{p}}$, to moderate confinement, $2 l_{\mathrm{p}}<h<6 l_{\mathrm{p}}$.

To explore the effect of an attractive dsDNA-surface interaction on $R_{\mathrm{g} \|}$, we consider an attractive polymer-surface interaction with strength $\varepsilon_{\mathrm{S}}=0.7$, which is smaller than the critical value for chain adsorption, $\varepsilon_{\mathrm{s}}{ }^{*}=0.75$ where $d=6.2 \mathrm{~nm}$ and $L=40 l_{\mathrm{p}}$. To explore the effect of an attractive dsDNA-dsDNA interaction, we select a polymer-polymer attractive interaction strength of $\varepsilon=0.2$, which is smaller than the critical value for chain collapse, $\varepsilon^{*}=0.44$ for chains having an effective diameter $d=6.2 \mathrm{~nm}$ and contour length $L=40 l_{\mathrm{p}}$. In Section 4 , we discuss experimental conditions relevant to these input parameters.

\section{Results and discussion of computation}

\subsection{Influence of chain diameter on swelling exponent}

We begin an analysis of our model by considering the radius of gyration of a chain in bulk solution. In Fig. 2, we show how the effective diameter $d$ affects the value of $R_{\mathrm{g}}$ of chains of the same contour length $L$ in bulk solution. Clearly, $R_{\mathrm{g}}$ increases as $d$ and the excluded volume interaction increase. We obtain the effective swelling exponent $\nu_{\text {bulk }}\left(d / l_{\mathrm{p}}\right)$ and the prefactor $A\left(d / l_{\mathrm{p}}\right)$ from a fit of the data in the main panel of Fig. 2 to a power law relation,

$$
R_{\mathrm{g}}\left(L / l_{\mathrm{p}}, d / l_{\mathrm{p}}\right)=A\left(\frac{L}{l_{\mathrm{p}}}\right)^{\nu_{\text {eff }}} l_{\mathrm{p}},
$$

where $A=A\left(d / l_{\mathrm{p}}\right)$ and $\nu_{\text {eff }}=\nu_{\text {eff }}\left(d / l_{\mathrm{p}}\right)$ are dimensionless parameters. The effective swelling exponent $\nu_{\text {eff varies by only } \approx 1 \%}$ over the range of contour lengths that we investigate, which is small in comparison to the effects of confinement, so we approximate $\nu_{\text {eff }}$ to be independent of $L$.

The blue solid triangle in the inset of Fig. 2 denotes recent MC calculations ${ }^{37}$ which assumed an effective diameter of $d=2.4 \mathrm{~nm}$. Again, we note that a variable reduction, performed by dividing $\nu, R_{\mathrm{g}}$ and $L$ by the persistence length $l_{\mathrm{p}}$, does not lead to a universal reduced variable description required by the worm-like chain model without excluded volume interactions.

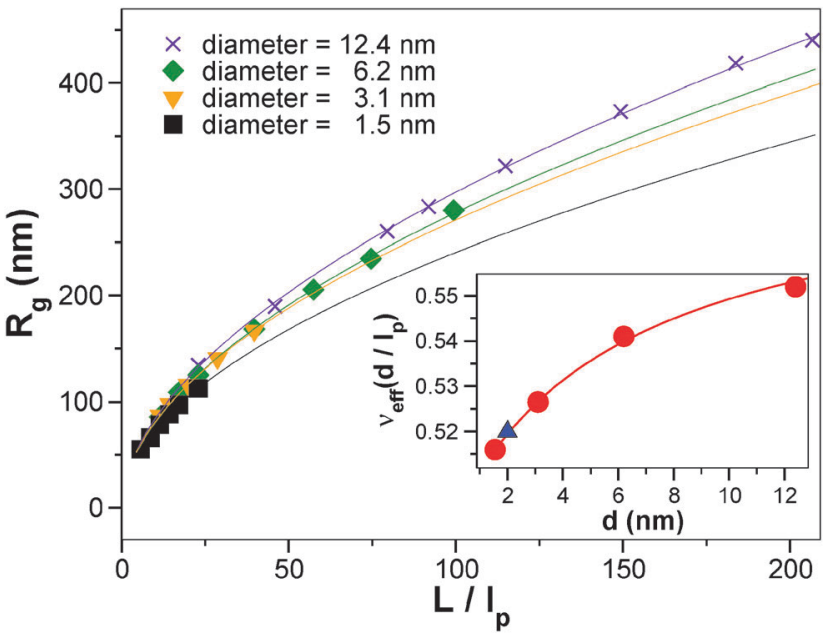

Fig. 2 The main panel shows the radius of gyration $R_{\mathrm{g}}$ as a function of contour length $L$ normalized by the persistence length $l_{p}$ for chains with different effective diameters $d$ in bulk solution. We fix the persistence length of the chain to $l_{p}=54 \mathrm{~nm}$ in all these calculations. The symbols are data obtained by MD simulations and the solid lines are fits to eqn (6). The inset shows the effective swelling exponent $\nu_{\text {bulk }}(d)$ as a function of $d$. The red circles are data obtained by MD simulations, and the blue solid triangle is data from Monte Carlo (MC) calculations. ${ }^{37}$ The red solid line is a fit to eqn (7).

Therefore, we dispense with this model for predicting the size of dsDNA under strong slit-like confinement. Instead, we calculate $R_{\mathrm{g}}$ for a range of effective diameters $d=(1.55,3.1,6.2$, and 12.4) $\mathrm{nm}$, and different contour lengths $L$ ranging from $\approx 10 l_{\mathrm{p}}$ to $\approx 200 l_{\mathrm{p}}$. The inset in Fig. 2 shows that $\nu_{\text {bulk }}\left(d / l_{\mathrm{p}}\right)$ increases with $d$. The solid line is given by the empirical relation,

$$
\nu_{\text {bulk }}\left(d / l_{\mathrm{p}}\right)=\nu_{\mathrm{RW}}+\Delta \nu^{*} \frac{d / d^{*}}{1+d / d^{*}},
$$

which describes this variation rather well, with a correlation coefficient $R^{2}=0.998$. Here, $\nu_{\mathrm{RW}}=0.5$ is the theoretical value for ideal chains, and the change in the swelling exponent $\Delta \nu^{*}=$ $0.08 \pm 0.001$ is the deviation of $\nu$ from this theoretical value. For chains with $d \gg d^{*}$, the effective swelling exponent $\nu_{\text {bulk }} \approx 0.58$ is that predicted for self-avoiding walks. ${ }^{22}$ This analysis indicates a crossover effective diameter $d^{*}=6.23 \pm 0.07 \mathrm{~nm}$, for $l_{\mathrm{p}}=54 \mathrm{~nm}$, at which the excluded volume interaction becomes appreciable even for dsDNA in bulk solution. Coincidentally, this value is similar to that suggested in recent experiments ${ }^{8,12}$ for dsDNA labeled with YOYO-1 dye. Using eqn (7) we predict $\nu_{\text {bulk }}(d)$ for a wide range of effective diameters in the following simulations. We find that the prefactor $A_{\text {bulk }}\left(d / l_{\mathrm{p}}\right)$ fluctuates slightly with $d$. Specifically, $A_{\text {bulk }}\left(d / l_{\mathrm{p}}\right)=(0.433 \pm 0.01,0.426 \pm 0.008,0.440 \pm$ $0.02,0.412 \pm 0.01)$ for effective diameters $d=(12.4,6.2,1.55,3.1)$ $\mathrm{nm}$, respectively.

\subsection{Influence of slit height on swelling exponent}

We now consider the main topic of simulating dsDNA confined to a nanofluidic slit. The upper panel of Fig. 3 shows the radius of gyration $R_{\mathrm{g}}$ as a function of contour length $L$ for chains in the transition between moderate and strong confinement. As expected, $R_{\mathrm{g}}$ increases as $h$ decreases. We fit the data from the 

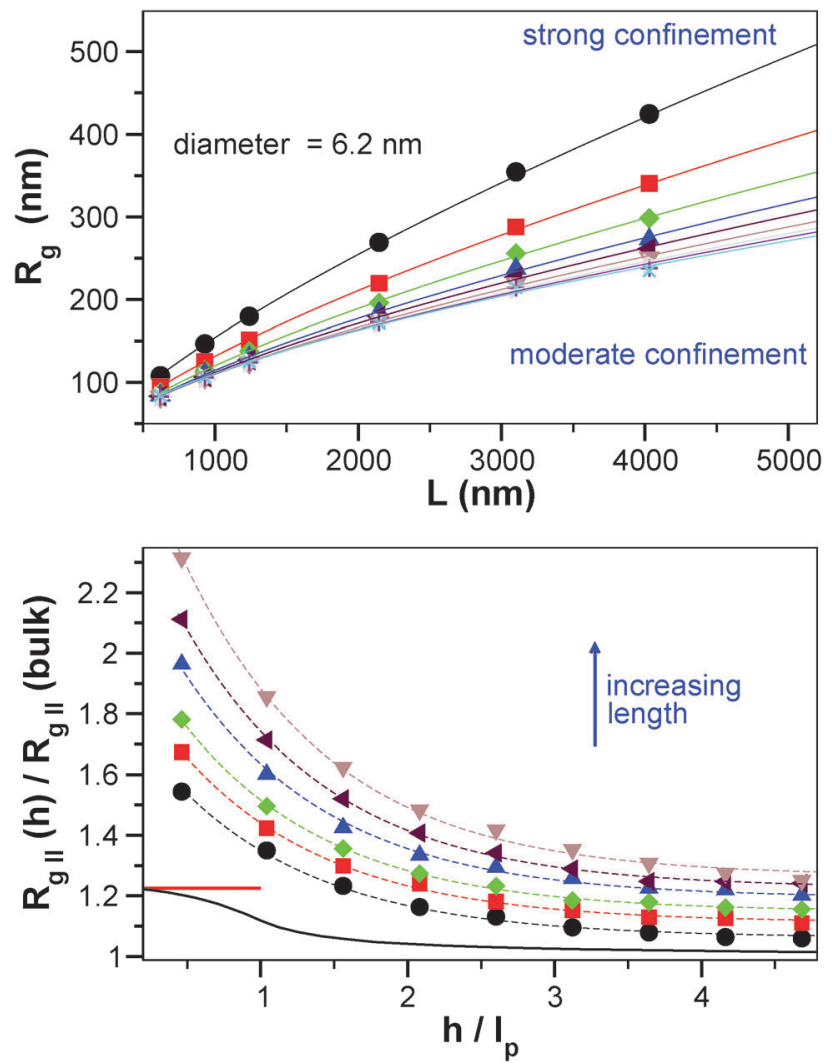

Fig. 3 The upper panel shows the radius of gyration $R_{\mathrm{g}}$ for a chain with a fixed effective diameter $d=6.2 \mathrm{~nm}$ as a function of contour length $L$ for chains ranging from $11.5 l_{p}$ to $75 l_{p}$ under confinement to a slit of height $h$. As expected, $R_{\mathrm{g}}$ increases as $h$ decreases. The symbols are the simulation results and the solid line is a fit to eqn (6). The lower panel shows the in-plane radius of gyration normalized by its bulk value $R_{\mathrm{g} \|}(h) / R_{\mathrm{g} \|}(\mathrm{bulk})$ as a function of the slit height $h$ normalized by the persistence length $l_{\mathrm{p}}$. The solid symbols represent the MD simulations and the dashed lines guide the eyes. The gray solid line is the result of $\mathrm{MC}$ calculations for an ideal random walk chain model, and the red solid line is the exact prediction for the change in chain size for a strongly confined random walk polymer, ${ }^{16}$ $R_{g \|}(h) / R_{g \|}($ bulk $)=\sqrt{3 / 2}$.

upper panel of Fig. 3 to eqn (6) to obtain $A$ and $\nu$ for confined chains. The correlation coefficients $R^{2}$ for all of the fits in this panel are $\geq 0.998$. As the surfaces of a nanofluidic slit strongly confine a dsDNA molecule, the spatial dimensions available to the molecule to explore decrease. We quantify the effect of this dimensional reduction by computing $\nu_{\mathrm{eff}}\left(d / l_{\mathrm{p}}, h / l_{\mathrm{p}}\right)$ over a range of $h$ that corresponds to the regimes of strong and moderate confinement in the lower panel of Fig. 1.

The upper panel in Fig. 4 shows the influence of confinement on the prefactor $A\left(d / l_{\mathrm{p}}, h / l_{\mathrm{p}}\right)$ and the lower panel shows the effective swelling exponent $\nu_{\mathrm{eff}}\left(d / l_{\mathrm{p}}, h / l_{\mathrm{p}}\right)$ for chains having different effective diameters: $d=(1.55,3.1$, and 6.2$) \mathrm{nm}$. The solid lines for the upper panel are described by,

$$
A\left(d / l_{\mathrm{p}}, h / l_{\mathrm{p}}\right)=\Delta A\left(d / l_{\mathrm{p}}\right)+\frac{0.14}{1+\exp \left(-h / l_{\mathrm{p}}+4.5\right)},
$$

with $\Delta A\left(d / l_{\mathrm{p}}\right)=(0.33 \pm 0.02,0.33 \pm 0.02,0.32 \pm 0.02)$ for $d=(1.55,3.1,6.2) \mathrm{nm}$ respectively. The solid lines in the lower
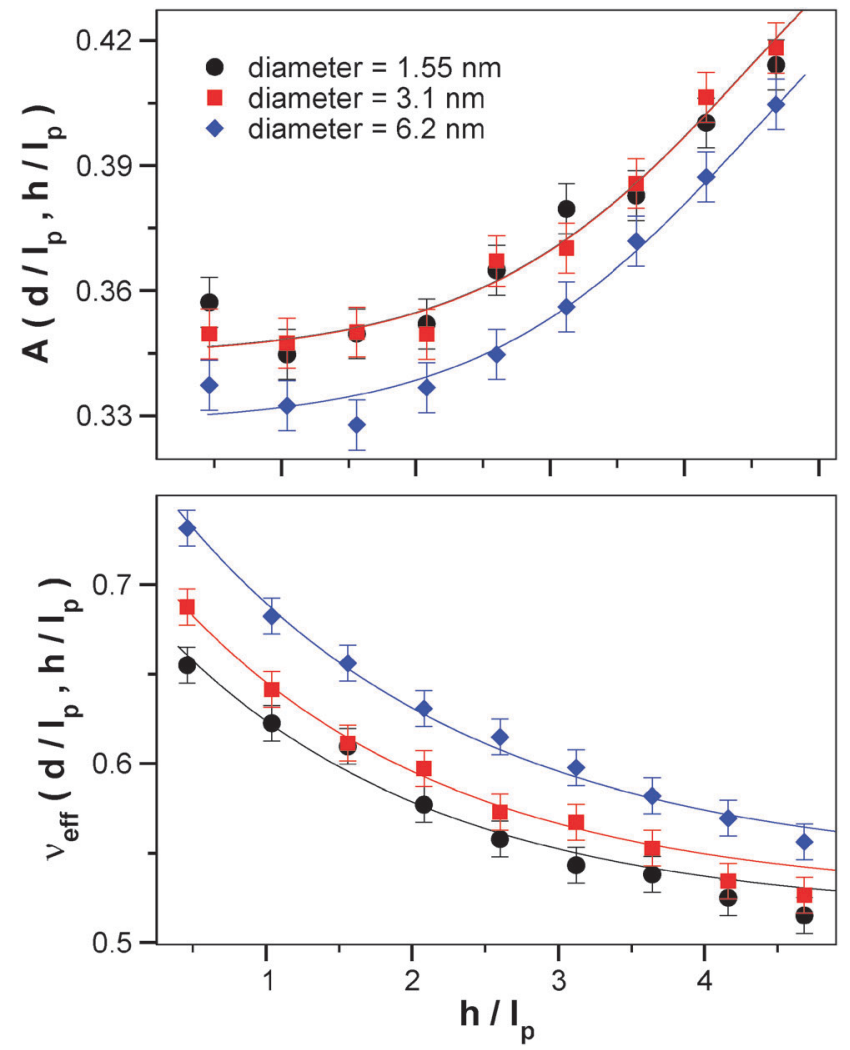

Fig. 4 The upper panel shows the prefactor $A$ and the lower panel shows the effective swelling exponent $\nu_{\text {eff }}$ for chains having different effective diameter $d$ as a function of the slit height $h$ normalized by the persistence length $l_{\mathrm{p}}$. The solid lines are fits to eqn (8) and (9), for $A$ and $\nu_{\text {eff, }}$ respectively. Both $A\left(d / l_{p}, h / l_{p}\right)$ and $\nu_{\text {eff }}\left(d / l_{p}, h / l_{p}\right)$ change with decreasing $h$.

panel are a fit to the effective swelling exponent $\nu_{\mathrm{eff}}\left(d / l_{\mathrm{p}}, h / l_{\mathrm{p}}\right)$ for the confined chains,

$$
\nu_{\text {eff }}\left(d / l_{\mathrm{p}}, h / l_{\mathrm{p}}\right)=\nu_{\text {bulk }}\left(d / l_{\mathrm{p}}\right)+\Delta \nu\left(d / l_{\mathrm{p}}\right) \exp \left(-h / h^{*}\right),
$$

where $\Delta \nu\left(d / l_{\mathrm{p}}\right)=(0.190 \pm 0.04,0.205 \pm 0.04,0.245 \pm 0.04)$, and $h^{*}=(1.730 \pm 0.05,1.820 \pm 0.06,2.070 \pm 0.05) \mathrm{nm}$ for $d=(1.55$, 3.2 or 6.2$) \mathrm{nm}$, respectively. Both $A(d, h)$ and $\nu_{\text {eff }}(d, h)$ change substantially with decreasing $h / l_{\mathrm{p}}$. The correlation coefficients $R^{2}$ for all of the fits in Fig. 4 are $\geq 0.97$.

To emphasize the importance of excluded volume interactions under strong confinement, we compare our MD simulation results to $\mathrm{MC}$ simulation results and renormalization group theory results in the lower panel of Fig. 3. We show the results of an off-lattice MC calculation of the mode value of the $R_{\mathrm{g} \|}$ distribution for a random walk chain of contour length $400 l_{\mathrm{p}}$ confined between two reflective boundaries. In this case, $R_{\mathrm{g} \|}$ changes only modestly. The solid red line is the exact prediction of the change in $R_{\mathrm{g} \|}$ for a random walk chain under strong confinement. ${ }^{16}$ The MC simulation results and the renormalization group theory results are in excellent agreement at the two-dimensional limit. ${ }^{16}$ These discrete random walk chains exhibit effects of dimensional reduction that do not exist for continuum Gaussian chains, but the changes are much smaller in this type of model than those in a model with excluded volume interactions. 
Cifra $^{10}$ performed MC calculations using a similar model, and his results are in qualitative agreement with ours. Hsu and Binder $^{39}$ performed MC calculations of semi-flexible polymer chains with substantial contour lengths, but the effective diameter could not be varied in these lattice calculations. Other simulations aimed at describing dsDNA under confinement, ${ }^{12}$ apart from $\mathrm{Cifra}^{10}$ and $\mathrm{Hsu}$ and Binder, ${ }^{39}$ have not made realistic assumptions about the effective diameter of dsDNA, as we discuss in Section 2.2 and Section 4.

\subsection{Influence of polymer-surface and polymer-polymer interactions on chain size under confinement}

So far, our simulations have focused on the confinement of semi-flexible chains with excluded volume interactions by two impenetrable boundaries, to model the steric interactions of dsDNA molecules under confinement to nanofluidic slits. However, dsDNA in solution has a propensity to adsorb onto surfaces, ${ }^{40,41}$ and measurements of second virial coefficients from X-ray scattering of bulk solutions of dsDNA suggest the existence of attractive dsDNA-surface and dsDNA-dsDNA interactions ${ }^{42}$ under experimentally relevant circumstances. It is then important to consider the relevance of attractive dsDNAsurface and dsDNA-dsDNA interactions to the size of confined dsDNA. We investigate these effects using the model described in Section 2.1. For specificity, we fix the contour length of the dsDNA to be $L=40 l_{\mathrm{p}}$ and the effective diameter $d=6.2 \mathrm{~nm}$.

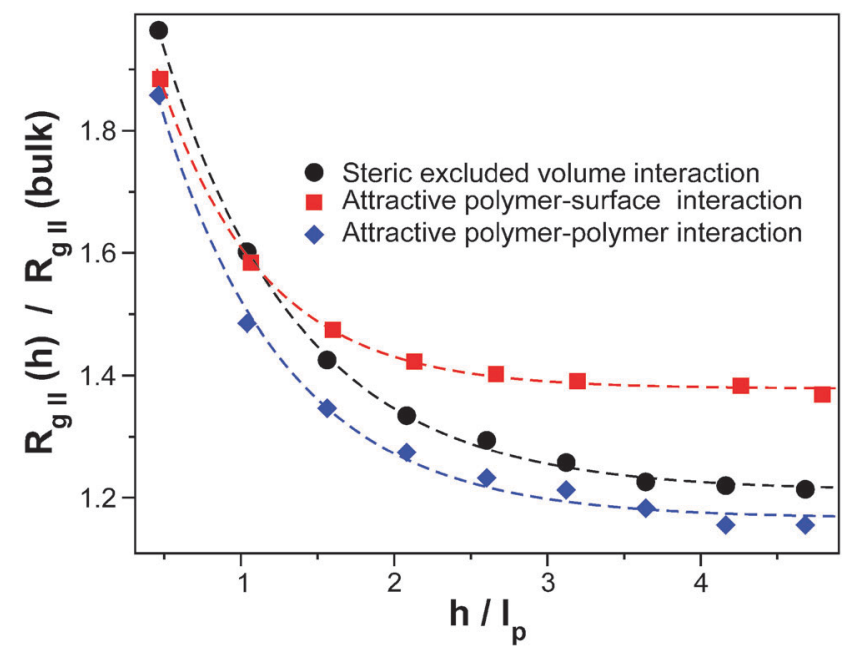

Fig. 5 This plot shows the in-plane radius of gyration normalized by the bulk solution value for chains having an effective diameter $d=6.2 \mathrm{~nm}$ and contour length $L=40 l_{p}$ as a function of slit height normalized by the persistence length, $h / l_{\mathrm{p}}$. The black circles are chains with excluded volume interactions in steric confinement, for reference. The red squares are chains with an attractive polymer-surface interaction, having a strength $\varepsilon_{\mathrm{s}}=0.7$ that is less than the interaction strength for adsorption, $\varepsilon_{\mathrm{s}}{ }^{\star}=0.75$. The effect of this interaction is appreciable and, can increase or decrease the size of the chain depending on the regime of confinement. The blue diamonds are chains with an attractive polymer-polymer interaction, having a strength $\varepsilon=0.2$ that is insufficiently attractive to cause chain collapse $\varepsilon^{\star}=0.44$. The effect of this interaction is always to decrease the size of the chain. Dashed lines guide the eyes.
We return to the discussion of the best estimate of the effective diameter in Section 4.

In Fig. 5, we first explore the effect on chain size of an attractive polymer-surface interaction in the presence of the excluded volume interaction under confinement. We find that the attractive interaction increases the chain size over a large range of $h / l_{\mathrm{p}}$ in moderate confinement, reducing the effective value of $h$ and enhancing the effect of dimensional reduction. In contrast, the transition to strong confinement terminates this enhancement. We next consider how an attractive polymerpolymer interaction influences the chain size. For an attractive interaction that is weak in comparison to the interaction strength at which chain adsorption occurs, the chain size decreases over the entire $h / l_{\mathrm{p}}$ range. In summary, we find that while attractive polymer-polymer and polymer-surface interactions can have an appreciable effect on the chain size, the effect of these interactions is of secondary importance in comparison to the dominant excluded volume interaction. Of course, this analysis applies only to a thermodynamic regime in which dsDNA is neither adsorbed onto the surface nor collapsed into a compact state.

This results in a complex interaction coupling problem, because the attractive polymer-surface and polymer-polymer interactions are competitive. Therefore, the adsorption and collapse transitions depend strongly on the strength of these interactions, as well as the effective diameter, persistence length, and contour length. We will investigate this coupling problem in the future. Here, we emphasize the main qualitative effects of these interactions, in relation to the following comparison of computational results and experimental measurements. Since these interactions are competitive, the strength of the interactions are unknown, and the experimental measurements do not show clear evidence of adsorbing or collapsing dsDNA molecules, we return to a model of the system considering only the steric confinement of model chains with excluded volume interactions.

\section{Comparison of computational results and experimental measurements}

\subsection{Quantitative comparison of computed and measured sizes}

To directly compare our computational results to the experimental measurements described in Section 1, we must solve a computational problem - it is not currently feasible to model dsDNA having a contour length $L \geq 400 l_{\mathrm{p}}$ using either MD or MC calculations. This is a practical concern, because the experimental studies have used lambda phage dsDNA as a reference sample. Human genomic dsDNA, is even longer. To solve this problem, we use our estimate of the change in $\nu$ and $A$ with dimensionality to predict the ratio $R_{\mathrm{gl|}}(h) / R_{\mathrm{g} \|}$ (bulk) for chains in the experimental regime. Fig. 6 shows $R_{\mathrm{g} \|}(h) / R_{\mathrm{g} \|}$ (bulk) for chains with different values of $d=1.55 \mathrm{~nm}$, $3.1 \mathrm{~nm}$ or $6.2 \mathrm{~nm}$ and $L=450 l_{\mathrm{p}}$. The computational results and the experimental measurements are in good agreement for effective diameters ranging from $d=1.55 \mathrm{~nm}$ to $3.1 \mathrm{~nm}$. 

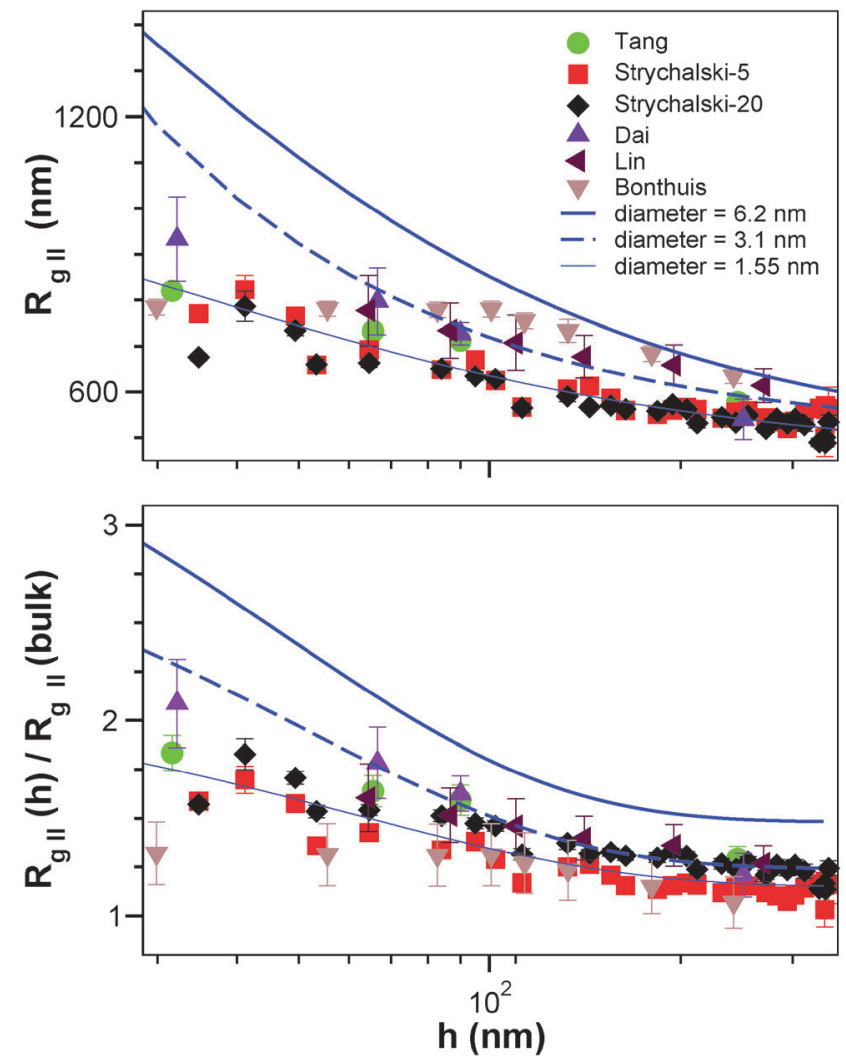

Fig. 6 The upper panel shows a comparison between the experimental measurements described in Section 1 and our computational results. The symbols are the experimental measurements and the blue lines are computational results for chains with different effective diameters $d$. The lower panel shows the upper panel data normalized by the in-plane radius of gyration in bulk solution. This normalization introduces additional uncertainties in the experimental results, systematically shifting the data. The computational results and the experimental measurements are in good agreement for effective diameters ranging from $d=1.55 \mathrm{~nm}$ to $3.1 \mathrm{~nm}$. This range is consistent with standard estimates from molecular biology of the effective diameter of dsDNA.

Molecular biology studies indicate that dsDNA exhibits three common forms: an anhydrous A-form, and a hydrated B-form that are both right-handed helical structures, and an exotic Z-form that forms a left-handed helix. The B-form is most common in solutions of dsDNA. Standard estimates of the bare diameter of those forms of dsDNA are $2.3 \mathrm{~nm}, 2.0 \mathrm{~nm}, 1.8 \mathrm{~nm}$, respectively. ${ }^{32}$ It is normal to add a hydration layer thickness of about $0.5 \mathrm{~nm}$ to these bare diameters, leading to an estimated effective diameter $d=2.4 \mathrm{~nm}$ for B-dsDNA in solution. However, we encounter experimental estimates of the effective diameter ranging from $d \approx 2.0 \mathrm{~nm}^{8}$ to $d \approx 27 \mathrm{~nm} \cdot{ }^{33,34}$ Stigter attributed the relatively large effective diameter to charges on the dsDNA chains. ${ }^{35}$ Smith and co-workers ${ }^{36}$ have recently argued that these large effective diameter estimates of $d$ are consistent with measurements of diffusion coefficients of dsDNA, but Mansfield and co-workers ${ }^{37,38}$ have found that a effective diameter of $d=2.4 \mathrm{~nm}$ provides a selfconsistent description of dsDNA self-diffusion, sedimentation coefficient, radius of gyration, and intrinsic viscosity, so that this effective diameter renormalization is apparently unwarranted.
The results of our comparative analysis indicate that the standard estimate suggested by molecular biology accurately models the effective diameter of dsDNA molecules under confinement to nanofluidic slits. This consistency supports our model of dimensional reduction and emphasizes the amplification of the excluded volume interaction of semi-flexible polymers under slit-like confinement. In the following subsections, we further connect our input parameters with the experimental systems and discuss additional experimental uncertainties that are outside the scope of our simple model.

\subsection{Fluorescent dyes}

Imaging dsDNA by fluorescence microscopy commonly involves labeling the molecules with bis-intercalating dimeric cyanine dye, resulting in a dsDNA-dye complex. This potentially alters the persistence length and effective diameter of native dsDNA, and nominally increases the contour length by as much as $50 \%$ (ref. 45). Of the experimental measurements discussed in Section 1, Strychalski et al. ${ }^{11}$ tested two dsDNA-YOYO-1 complexes with different stoichiometries, discussed related uncertainties in actual numbers of dye molecules, and concluded that a decreased persistence length or effective diameter might have compensated for an increased contour length. Other measurements focused on this topic have indicated that the persistence length is invariant to the presence of YOYO-1 at equilibrium. ${ }^{43,44}$ Gel chromatography and sedimentation mobility of dsDNA molecules under similar experimental conditions have also been insensitive to the presence of the dye, suggesting little change in the effective diameter. ${ }^{36}$ In any case, imaging measurements with molecular resolution would help to understand exactly how the dye influences the structure of the dsDNA, but at present we conclude that any effect of the dye on the persistence length and effective diameter of dsDNA is of secondary importance. Considering these various issues, we use a nominal value of $l_{\mathrm{p}}=54 \mathrm{~nm}$ and a representative value of $L=21.6 \mu \mathrm{m}$ as input parameters in our computations.

\subsection{Measurements and inferences of dsDNA size}

Direct imaging measurements of $R_{\mathrm{g} \|}$ in bulk solution, for normalizing measurements of $R_{\mathrm{g} \|}$ under confinement to nanofluidic slits, are inherently uncertain. In particular, the diffusion of segments of an anisotropic dsDNA molecule above and below the depth of field of an imaging system introduces measurement errors that are difficult to quantify. Such measurements are then less accurate than direct imaging measurements of dsDNA molecules under confinement to nanofluidic slits with heights that are less than or equal to the depth of field. In this case, direct imaging errors due to optical diffraction and camera pixilation increase the apparent size of dsDNA molecules by $<10 \%$, as Strychalski et al. ${ }^{11}$ estimated and corrected by simulation. Table 1 reports measurement parameters of the imaging systems relevant to this analysis. In an alternate approach to avoiding these direct imaging errors, the value of $R_{\mathrm{g} \|}$ in bulk solution reported by Tang et al. ${ }^{8}$ and Dai et al. ${ }^{12}$ was an indirect inference from a diffusivity measurement as an input to a modified Stokes-Einstein equation, while the 
confined dsDNA sizes estimates by Tang were measured by direct imaging. In particular, Dai et al. ${ }^{12}$ inferred $R_{\mathrm{g} \|}$ from diffusivity measurements as inputs into a blob scaling argument using an assumed relationship between $R_{\mathrm{H}}$ and $R_{\mathrm{g}}$. This inference of dsDNA size introduces uncertainties that are difficult to quantify, and we briefly discuss this problem.

One of the many problems in inferring $R_{\mathrm{g} \|}$ in bulk solution from a hydrodynamic model is the assumption that the radius of gyration $R_{\mathrm{g}}$ scales in direct proportion to the hydrodynamic radius $R_{\mathrm{H}} \cdot{ }^{37}$ This scaling ansatz incorrectly assumes that hydrodynamic interactions among segments of a dsDNA molecule are infinitely strong. ${ }^{37}$ By implication, $R_{\mathrm{H}}$ scales with contour length $L$ differently than $R_{\mathrm{g}}$ for the contour length and range of ionic strengths in the dsDNA measurements discussed in Section 1. In particular, the effective swelling exponent for $R_{\mathrm{g}}$ of dsDNA in bulk solution is $\nu \approx 0.52$, as we discussed in Section 2 , but the effective scaling exponent for $R_{\mathrm{H}}$ is $\nu_{\mathrm{H}} \approx 0.6 .{ }^{37}$ In summary, $R_{\mathrm{g}}$ and $R_{\mathrm{H}}$ are inherently different measures of polymer size. ${ }^{37,38}$

Scattering measurements of $R_{\mathrm{g}}$ for massive macromolecules are inherently difficult, ${ }^{36,45}$ motivating novel imaging measurements to estimate the value of $R_{\mathrm{g} \|}$ in bulk solution. For example, more reliable estimates of $R_{\mathrm{g} \|}$ could be made by tethering one end of a dsDNA molecule to a non-adsorbing surface to limit the diffusion of molecule. ${ }^{36}$ Calculation by renormalization group methods of $R_{\mathrm{g}}$ for random walk chains and and chains with excluded volume indicate that end-tethering should only slightly perturb $R_{\mathrm{g}},{ }^{46}$ on the order of an increase of $3 \%$ from bulk solution, provided the chains are not adsorbed. ${ }^{47}$

\subsection{Solution conditions}

Tang et $a l .{ }^{8}$ and Strychalski et $a .^{11}$ have discussed differences between the buffer systems. We extend this discussion within the framework of our model. Several of the buffer solutes have been implicated in causing variations in dsDNA size. Monovalent cations of both conjugate acids and electrolytes tend to associate with dsDNA. ${ }^{48}$ The interaction of $\mathrm{Na}^{+}$with dsDNA is similar to but weaker than that of the acidic form of Tris $\left(\right.$ Tris $\left.^{+}\right),{ }^{49}$ which was present in all of the experimental systems. Therefore, the presence of $\mathrm{Na}^{+}$in the experiments of Bonthuis et $a .^{6}{ }^{6}$ and Lin et $a .^{13}$ probably did not significantly influence the dsDNA size. With regard to anions, however boric acid probably did significantly influence the dsDNA size. ${ }^{50}$ In particular, Tang et al. ${ }^{8}$ found that the absence of boric acid in the experimental system of Bonthuis et $a .^{6}{ }^{6}$ resulted in moderate changes in dsDNA size relative to the other experimental systems. ${ }^{8,11-13}$

The various oxygen scavenging additives used to mitigate the photobleaching of YOYO- 1 and the photodegradation of dsDNA are also a concern. In the presence of dissolved oxygen, the enzymatic oxygen scavenging system comprised of glucose, glucose oxidase, and catalase produced gluconic acid $\left(\mathrm{p} K_{\mathrm{a}} \approx\right.$ 3.86). This may have reduced the $\mathrm{pH}$ of the buffer system and change the dsDNA size. ${ }^{51}$ Even at saturated levels of dissolved oxygen at room temperature and pressure $\left(\approx 9 \mathrm{mg} \mathrm{mL}^{-1}\right)$, however, only $\approx 0.3 \mathrm{mmol} \mathrm{L}^{-1}$ solution of gluconic acid would have been produced which is $<1 \%$ of the buffer capacity of
Tang et $a .^{8}$ and Lin et al. ${ }^{13}$ The chemical oxygen scavenging system of $\beta$-mercaptoethanol had a weak reducing capability $(0.26 \mathrm{~V}$ at $\mathrm{pH}=7)$ and is not expected to have directly influenced dsDNA size. As a weak acid $\left(\mathrm{p} K_{\mathrm{a}} \approx 9.63\right)$, however, $\beta$-mercaptoethanol significantly contributed to the buffer at the concentrations used by Tang et al. ${ }^{8}\left(\approx 570 \mathrm{mmol} \mathrm{L}^{-1}\right)$, Strychalski et al. ${ }^{11}$ $\left(\approx 430 \mathrm{mmol} \mathrm{L}^{-1}\right)$, and Lin et al. ${ }^{13}\left(\approx 430 \mathrm{mmol} \mathrm{L}{ }^{-1}\right)$. The addition of $\beta$-mercaptoethanol probably increased the $\mathrm{pH}$ of the experimental buffer systems above that expected for a buffer system comprised only tris base, boric acid, and ethylenediaminetetraacetic acid. ${ }^{11}$ This may have influenced the dsDNA-dsDNA interaction. We conclude that there is a clear need for tighter control and better characterization of buffer chemistry and solution additives.

\section{5 dsDNA-surface interactions}

Non-steric interactions between dsDNA molecules and the surfaces of nanofluidic slits have been mentioned, but not substantiated, as having a potential influence on dsDNA size. ${ }^{13}$ Here, we explore this issue in greater detail. For nanofluidic slits with fused silica surfaces, dsDNA molecules interact with the slit surfaces by silanol groups $(\mathrm{SiOH})$, which cover the slit surfaces at a density ${ }^{5,52}$ of (4 to 5 ) $\mathrm{nm}^{-2}$. In the case of planar fused silica, there are three categories of silanol groups. Isolated silanols deprotonate freely $\left(\mathrm{p} K_{\mathrm{a}} \approx 4.9\right)$ to form SiO and comprise $\approx 19 \%$ of the silanol population. ${ }^{53}$ Geminal and vicinal silanols deprotonate under more basic conditions $\left(\mathrm{p} K_{\mathrm{a}} \approx 8.5\right)$ and comprise $\approx 81 \%$ of silanol population. ${ }^{53}$ Through a variety of mechanisms, ${ }^{54-57}$ protonated silanols interact attractively with the solution constituents. For relatively simple electrolyte buffers, such as those used by Bonthuis et $a .^{6}$ and Strychalski et al. ${ }^{11}$ the $\mathrm{pH}$ of the solution strongly influenced the value of $\varepsilon_{\mathrm{s}}{ }^{54,55,57}$ More acidic buffer systems result in slit surfaces enriched with protonated silanols and depleted in negative charge. Both effects increase $\varepsilon_{\mathrm{s}}$. In particular, the 12-base single-stranded segments at both $5^{\prime}$ ends of double-stranded lambda phage DNA, also known as sticky ends, interact attractively with the protonated silanols of planar fused silica surfaces. ${ }^{40}$ If present, single-stranded segments in locally melted regions of double-stranded dsDNA also interact attractively with protonated silanols. ${ }^{55}$ Bonthuis et al. ${ }^{6}$ did not report the $\mathrm{pH}$ of the buffer system used, but we infer this as $\mathrm{pH} \approx 8$ from the study of Ren and Stein, ${ }^{17,18}$ which used methods that "followed those described by Bonthuis et al." ${ }^{, 6}$ The actual pH of the solution of Bonthuis et al. ${ }^{6}$ may also have been lower, depending on the concentration of ethylenediaminetetraacetic acid used. With access to a significant number of protonated silanols at $\mathrm{pH}<8.5$, single-stranded segments of dsDNA may have interacted attractively with the slit surfaces of Bonthuis et al. ${ }^{6}$ to a significant extent. In contrast, Strychalski et al. ${ }^{11}$ used a buffer system that was sufficiently basic $(\mathrm{pH}=8.7)$ to have reduced the concentration of protonated silanols and suppressed attractive surface interactions. In addition, the presence of boric acid in the buffer system of Strychalski et al. ${ }^{11}$ resulted in dsDNA molecules with increased negative charge density and electrostatic repulsion from the slit surfaces. Alternatively, more complex buffer systems 
suppressed attractive surface interactions by masking surface silanols through the competitive nonspecific adsorption of other soluble species, such as proteins, glucose oxidase and catalase, as used by Tang et al., ${ }^{8}$ Dai et al., ${ }^{12}$ and Lin et al., ${ }^{13}$ as well as in proprietary blends of polymers and surfactants such as POP-6, as used by Lin et al. ${ }^{13}$ The quantitative effects of these diverse experimental conditions on dsDNA size are not known, however, our model indicates the possible relevance of these effects.

\subsection{Hydrodynamic flow}

Hydrodynamic flow significantly changes the behavior of dsDNA under confinement to nanofluidic slits. It is difficult to realistically model the combined effects of confinement and flow, but Brownian dynamics simulations of long chains in a microchannel with flow have provided some insight into this problem. ${ }^{58,59}$ Hydrodynamic polymer-surface interactions substantially distort the chains, even in the simple case that the thermodynamic polymer-surface interactions are repulsive. Other simulations and measurements have indicated that hydrodynamic polymer-polymer interactions due to a steady flow significantly distort the chain. ${ }^{59,60}$ Even without fluid flow, simulations have found a strong influence of hydrodynamic polymer-surface interactions on the diffusion of confined dsDNA. ${ }^{61}$ Bonthuis et $a l .{ }^{6}$ reported residual fluid flows of less than $1 \mu \mathrm{m} \mathrm{s}^{-1}$, resulting in hydrodynamic interactions that our model does not treat. We conclude that it is not appropriate to directly compare these experimental measurements with hydrodynamic flow to experimental measurements and computational results at thermodynamic equilibrium.

\section{Conclusions}

The inconsistency of recent measurements by fluorescence microscopy of single dsDNA molecules under confinement to nanofluidic slits has motivated our use of a simple model to explore the factors that might explain this variability. Our computational results indicate that strong confinement highly amplifies polymer excluded volume interactions through dimensional reduction of the system. These interactions, which have a limited significance in bulk solution, become highly relevant in a nanofluidic slit. Therefore, greater experimental control of factors influencing the excluded volume interaction is necessary to improve experimental reproducibility. Attractive polymer-surface and polymer-polymer interactions can also influence the dsDNA size appreciably, but the polymer-surface interaction only has a limited effect under non-adsorbing conditions. Our computational results are qualitatively consistent with previous simulations and theoretical arguments for semiflexible polymers under confinement ${ }^{20,62,63}$ that show similar amplification of excluded volume interactions.

Importantly, our computational analysis is novel in its introduction of quantitative swelling exponents and prefactors that vary with the scale of confinement, allowing the prediction of the behavior of long dsDNA molecules that are not currently accessible by state-of-the-art MD simulations. This data reduction scheme is important not only for assessing the experimental measurements of concern, but also for predicting the behavior of even longer molecules, such as human genomic dsDNA, under nanoscale confinement. However, such predictions will be sensitive to the input value of the effective diameter. Our computational methodology can be generalized for other types of polymers with different values of persistence length.

Based on insights from our simulations, we assess corresponding experimental measurements on confined duplex DNA with an aim of informing how to better control the relevant experimental variables for future measurements. The measurements of Tang et al., ${ }^{8}$ Strychalski et al. ${ }^{11}$ Lin et al.,${ }^{13}$ and Dai et al. ${ }^{12}$ are all qualitatively consistent with each other and our computational results, although there are influences related to solvent quality and polymer-surface interactions that clearly need to be under better control to improve experimental reproducibility.

While our simulations have focused on linear dsDNA under confinement to nanofluidic slits, other chain topologies and confinement schemes are of interest for future study. For example, circular dsDNA, which has been studied much less in experiments but is nonetheless highly relevant to various applications, has shown interesting trends in size variation under confinement to nanofluidic slit. In particular, the combination of nanofluidic devices with high confinement resolution around the transition from strong to moderate confinement, ${ }^{64}$ with an empirical, statistical analysis ${ }^{65}$ identified a localized influence of topology on the size variation of circular dsDNA at a slit height of $\approx 3 l_{\mathrm{p}}$. The same analysis did not resolve a similar transition for linear dsDNA, but such a transition remains a possibility. These observations motivate simulations to discern the underlying physical mechanisms, as well as the development of nanofluidic devices with even higher confinement resolution around these critical slit heights. Such devices would also be relevant for studying and applying nonequilibrium dsDNA dynamics induced by complex slit-like confinement schemes. ${ }^{66}$

Finally, we note that many measurements and applications of dsDNA under strong confinement conditions involve rectangular channels or cylindrical pores. The effect of dimensionality reduction should have an even larger effect for these geometries, since $\nu$ equals 1 for one-dimensional semi-flexible polymers. Consistent with this expectation, dsDNA molecules swell significantly in this type of channel where the magnitude of the effect is sensitive to the salt concentration. ${ }^{67}$ In the future, the same methodology of the present article can apply to study these more extreme cases of confinement.

\section{Disclaimer}

This article identifies certain commercial materials, equipment, or instruments to specify experimental procedures. Such identification implies neither recommendation or endorsement by the National Institute of Standards and Technology nor that the materials or equipment identified were necessarily the best available for the purpose. 


\section{Acknowledgements}

This work was supported in part by NIST award 70NANB13H202.

\section{References}

1 A. Milchev and K. Binder, Eur. Phys. J. B, 1998, 3, 477-484.

2 H.-P. Hsu and P. Grassberger, J. Chem. Phys., 2004, 120, 2034-2041.

3 P.-K. Lin, C.-C. Fu, Y.-L. Chen, Y.-R. Chen, P.-K. Wei, C. Kuan and W. Fann, Phys. Rev. E: Stat., Nonlinear, Soft Matter Phys., 2007, 76, 011806.

4 D. I. Dimitrov, A. Milchev, K. Binder, L. I. Klushin and A. M. Skvortsov, J. Chem. Phys., 2008, 128, 234902.

5 E. A. Strychalski, S. M. Stavis and H. G. Craighead, Nanotechnology, 2008, 19, 315301.

6 D. Bonthuis, C. Meyer, D. Stein and C. Dekker, Phys. Rev. Lett., 2008, 101, 108303.

7 T. Odijk, Phys. Rev. E: Stat., Nonlinear, Soft Matter Phys., 2008, 77, 060901.

8 J. Tang, S. L. Levy, D. W. Trahan, J. J. Jones, H. G. Craighead and P. S. Doyle, Macromolecules, 2010, 43, 7368-7377.

9 D. Chaudhuri and B. Mulder, Phys. Rev. E: Stat., Nonlinear, Soft Matter Phys., 2011, 83, 031803.

10 P. Cifra, J. Chem. Phys., 2012, 136, 024902.

11 E. A. Strychalski, J. Geist, M. Gaitan, L. E. Locascio and S. M. Stavis, Macromolecules, 2012, 45, 1602-1611.

12 L. Dai, J. J. Jones, J. R. C. van der Maarel and P. S. Doyle, Soft Matter, 2012, 8, 2972.

13 P.-k. Lin, C.-C. Hsieh, Y.-L. Chen and C.-F. Chou, Macromolecules, 2012, 45, 2920-2927.

14 C. Micheletti and E. Orlandini, Macromolecules, 2012, 45, 2113-2121.

15 K. D. Dorfman, D. Gupta, A. Jain, A. Muralidhar and D. R. Tree, Eur. Phys. J.: Spec. Top., 2014, 223, 3179-3200.

16 J. F. Douglas and K. F. Freed, Macromolecules, 1997, 9297, 1813-1817.

17 Y. Ren and D. Stein, Phys. Rev. Lett., 2011, 106, 068302.

18 D. Stein, Phys. Rev. Lett., 2011, 107, 049901.

19 K. F. Freed, J. Dudowicz, E. B. Stukalin and J. F. Douglas, J. Chem. Phys., 2010, 133, 094901.

20 M. Daoud and P. G. De Gennes, J. Phys. (Paris), 1977, 38, 85-93.

21 T. Odijk, Macromolecules, 1983, 16, 1340-1344.

22 K. F. Freed, Renormalization Group Theory of Macromolecules, Wiley, 1987.

23 H. Yamakawa, Modern theory of polymer solutions, Harper \& Row, 1971.

24 P. de Gennes, Scaling Concepts in Polymer Physics, Cornell University Press, 1979.

25 J. F. Douglas, B. J. Cherayil and K. F. Freed, Macromolecules, 1985, 18, 2455-2463.

26 J. F. Douglas, T. Ishinabe, A. M. Nemirovsky and K. F. Freed, J. Phys. A: Math. Gen., 1993, 26, 1835.

27 J. Cloizeaux and G. Jannink, Polymers in Solution: Their Modelling and Structure, OUP Oxford, 2010.
28 R. J. Baxter, Exactly Solved Models in Statistical Mechanics, Academic Press, London, 1982.

29 K. Kremer and G. S. Grest, J. Chem. Phys., 1990, 92, 5057-5086.

30 S. Plimpton, J. Comput. Phys., 1995, 117, 1-19.

31 D.-W. Yin, F. Horkay, J. F. Douglas and J. J. de Pablo, J. Chem. Phys., 2008, 129, 154902.

32 A. Rich, A. Nordheim and A. H. J. Wang, Annu. Rev. Biochem., 1984, 53, 791-846.

33 V. V. Rybenkov, N. R. Cozzarelli and A. V. Vologodskii, Proc. Natl. Acad. Sci. U. S. A., 1993, 90, 5307-5311.

34 N. M. Toan and C. Micheletti, J. Phys.: Condens. Matter, 2006, 18, S269.

35 D. Stigter, Biopolymers, 1977, 16, 1435-1448.

36 R. M. Robertson, S. Laib and D. E. Smith, Proc. Natl. Acad. Sci. U. S. A., 2006, 103, 7310-7314.

37 M. L. Mansfield and J. F. Douglas, Soft Matter, 2013, 9, 8914.

38 M. L. Mansfield, A. Tsortos and J. F. Douglas, Persistent Draining Crossover in DNA and Other Semi-Flexible Polymers: Evidence from Hydrodynamic Models and Extensive Measurements on DNA Solutions, J. Chem. Phys., 2015, to appear.

39 H.-P. Hsu and K. Binder, Macromolecules, 2013, 46, 8017-8025. 40 S. Isailovic, H.-W. Li and E. S. Yeung, J. Chromatogr. A, 2007, 1150, 259-266.

41 E. Ercolini, F. Valle, J. Adamcik, G. Witz, R. Metzler, P. De Los Rios, J. Roca and G. Dietler, Phys. Rev. Lett., 2007, 98, 058102 .

42 X. Qiu, K. Andresen, L. Kwok, J. Lamb, H. Park and L. Pollack, Phys. Rev. Lett., 2007, 99, 038104.

43 C. Zhang, F. Zhang, J. a. van Kan and J. R. C. van der Maarel, J. Chem. Phys., 2008, 128, 225109.

44 B. Kundukad, J. Yan and P. S. Doyle, Soft Matter, 2014, 10, 9721-9728.

45 J. Harpst and J. Dawson, Biophys. J., 1989, 55, 1237-1249.

46 M. Adamuti-Trache, W. E. McMullen and J. F. Douglas, J. Chem. Phys., 1996, 105, 4798.

47 J. F. Douglas, A. M. Nemirovsky and K. F. Freed, Macromolecules, 1986, 19, 2041-2054.

48 E. Stellwagen, Q. Dong and N. C. Stellwagen, Biopolymers, 2005, 78, 62-68.

49 Q. Dong, E. Stellwagen and N. C. Stellwagen, Biochemistry, 2009, 48, 1047-1055.

50 N. C. Stellwagen, C. Gelfi, P. G. Righetti and I. Cnr, Biopolymers, 2000, 54, 137-142.

51 X. Shi, J. Lim and T. Ha, Anal. Chem., 2010, 82, 6132-6138.

52 R. Iler, The Chemistry of Silica: Solubility, Polymerization, Colloid and Surface Properties and Biochemistry of Silica, Wiley, 1979.

53 S. Ong, X. Zhao and K. B. Eisenthal, Chem. Phys. Lett., 1992, 191, 327-335.

54 J. F. Allemand, D. Bensimon, L. Jullien, a. Bensimon and V. Croquette, Biophys. J., 1997, 73, 2064-2070.

55 P. E. Vandeventer, J. S. Lin, T. J. Zwang, A. Nadim, M. S. Johal and A. Niemz, J. Phys. Chem. B, 2012, 116, 5661-5670.

56 S. H. Kang, M. R. Shortreed and E. S. Yeung, Anal. Chem., 2001, 73, 1091-1099. 
57 K. a. Melzak, C. S. Sherwood, R. F. Turner and C. a. Haynes, J. Colloid Interface Sci., 1996, 181, 635-644.

58 R. M. Jendrejack, J. J. de Pablo and M. D. Graham, J. Chem. Phys., 2002, 116, 7752.

59 R. M. Jendrejack, D. C. Schwartz, M. D. Graham and J. J. de Pablo, J. Chem. Phys., 2003, 119, 1165.

60 T. T. Perkins, D. E. Smith, R. G. Larson and S. Chu, Science, 1995, 268, 83-87.

61 Y.-L. Chen, M. Graham, J. de Pablo, G. Randall, M. Gupta and P. Doyle, Phys. Rev. E: Stat., Nonlinear, Soft Matter Phys., 2004, 70, 060901.
62 J. H. van Vliet and G. ten Brinke, J. Chem. Phys., 1990, 93, 1436-1441.

63 J. H. Van Vliet, M. C. Luyten and G. Ten Brinke, Macromolecules, 1992, 25, 3802-3806.

64 S. M. Stavis, E. A. Strychalski and M. Gaitan, Nanotechnology, 2009, 20, 165302.

65 E. A. Strychalski, S. M. Stavis and J. Geist, AIP Adv., 2013, 3, 1-6. 66 S. M. Stavis, J. Geist, M. Gaitan, L. E. Locascio and E. A. Strychalski, Lab Chip, 2012, 12, 1174-1182.

67 W. Reisner, J. Beech, N. Larsen, H. Flyvbjerg, A. Kristensen and J. Tegenfeldt, Phys. Rev. Lett., 2007, 99, 058302. 